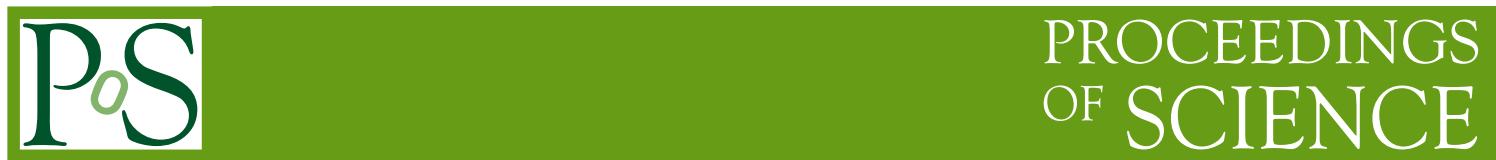

\title{
Narrow or Wide Resonance?
}

\section{T. Mart ${ }^{* \dagger}$}

Departemen Fisika, FMIPA, Universitas Indonesia, Depok 16424, Indonesia

E-mail: tmartefisika.ui.ac.id

We have investigated the existence of the $J^{p}=1 / 2^{+}\left(P_{11}\right)$ narrow resonance predicted by the chiral soliton model. For this purpose we used an isobar model developed for explaining the kaon photoproduction process. It was found that the most possible narrow resonance has a mass of $1650 \mathrm{MeV}$ and a width of $5 \mathrm{MeV}$. This finding is, however, different from that obtained in the $\eta$ photoproduction off a neutron, where the extracted mass and width are relatively larger, i.e. 1680 and $30 \mathrm{MeV}$, respectively. We have revisited the calculation by considering a wider resonance width and using the latest covariant isobar model. The result still indicates the existence of the narrow resonance with the mass of $1650 \mathrm{MeV}$, but the best agreement between experimental data and model prediction could be achieved by using the resonance mass and width of 1690 and 75 $\mathrm{MeV}$, respectively. However, the possibly small $N^{*}(1710) P_{11}$ coupling constant would constrain the mass and width to be $\lesssim 1680$ and $\lesssim 35 \mathrm{MeV}$, respectively.

XV International Conference on Hadron Spectroscopy

$4-8 / 11 / 2013$

Nara, Japan

\footnotetext{
* Speaker.

${ }^{\dagger}$ Research supported by Universitas Indonesia.
} 


\section{Introduction}

The nonstrange $P_{11}$ resonance plays a decisive role in the calculation of the masses of all antidecuplet baryon members predicted by the chiral quark soliton model $(\chi \mathrm{QSM})$ [1]. For instance, in estimating the pentaquark mass, Diakonov et al [1] assigned the non-strange $P_{11}$ resonance to the $N^{*}(1710) P_{11}$ listed by the Particle Data Group (PDG) [2], because at the time the PDG reported the resonance partial decay widths similar to those predicted by the $\chi \mathrm{QSM}$.

By using the masses of exotic baryons as inputs they reevaluated the mass of this resonance and found that it should be either 1647 or $1690 \mathrm{MeV}$, depending whether or not the mixing with lower-lying nucleonlike octet is excluded [3]. By definition, the resonance was expected to have a small total width, namely it must be a narrow resonance. This was also the reason that the small bump found in the $\eta$ photoproduction off a free neutron was suspected as an evidence of this resonance [ 4 ]. Lately, it is listed by the PDG as a new state with one-star status in the 2012 Review of Particle Physics [5] as the $N^{*}(1685)$, despite its spin and parity are still undetermined.

In this paper we report on some progress made in the investigation of this narrow resonance. Our discussion will be mainly based on our recent works published elsewhere [6, 元].

\section{Isobar Models}

In the first analysis we used the isobar model consisting of the standard $s-, u$-, and $t$-channel Born terms [6, 8]. Except for the nucleon resonances and contact terms, the corresponding Feynman diagrams are shown in Fig. 1. For the resonance terms the model exploits the Breit-Wigner multipole parameterization. The model was fitted to the low energy, near threshold, data. Detailed information on this model can be found in Refs. [6, 8].

In the second analysis we used the fully covariant isobar model [9]. This model consists of the standard $s^{-}, u$, and $t$-channel Born terms as well as the $K^{*+}(892), K_{1}(1270)$ vector mesons and the $\Lambda^{*}(1800) S_{01}, \Lambda^{*}(1810) P_{01}$ hyperon resonance. In the $s$-channel the model includes the $N^{*}(1650) S_{11}, N^{*}(1700) D_{13}, N^{*}(1710) P_{11}, N^{*}(1720) P_{13}, N^{*}(1840) P_{11}, N^{*}(1900) P_{13}, N^{*}(2080) D_{13}$, $N^{*}(2090) S_{11}$, and $N^{*}(2100) P_{11}$ nucleon resonances. These nucleon resonances are found to be consistent with the 2012 PDG listing [5]. The unitarity corrections are approximated by using the energy-dependent widths along with the appropriate partial branching fractions in the resonance propagators [10]. Since hadrons are composite objects, hadronic form factors are considered in hadronic vertices, where the gauge invariance of the amplitude after the form factor inclusion is

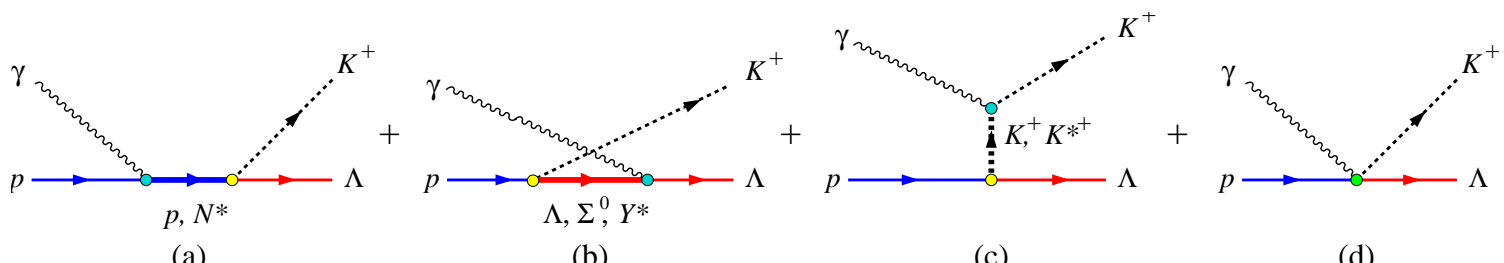

(a)

(b)

(c)

(d)

Figure 1: Feynman diagrams for the isobar model. Note that the diagram (d), called the contact or seagull diagram, is included to maintain gauge invariance of the amplitude after the inclusion of hadronic form factors [11]. 


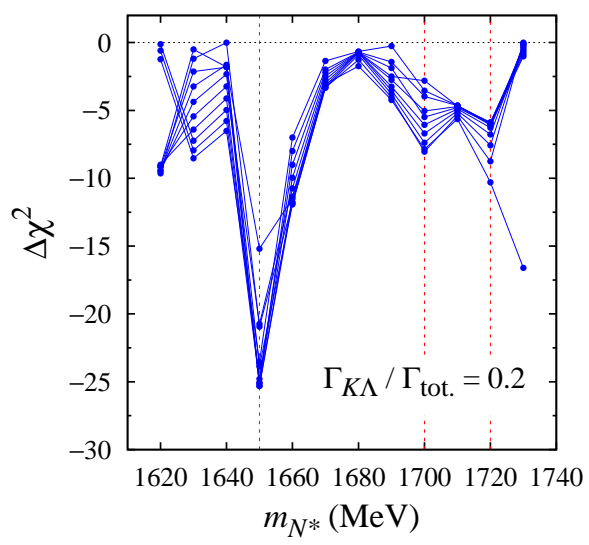

Figure 2: Changes of the $\chi^{2}$ in the fit of the kaon photoproduction data due to the inclusion of a narrow $P_{11}$ resonance. The negative value of $\Delta \chi^{2}$ indicates an improvement. The vertical lines locate the positions of $m_{N^{*}}=1650,1700$ and $1720 \mathrm{MeV}$.

restored by adding the contact term shown in Fig. 1](d) and using the Haberzettl prescription [11]. The model was fitted to the latest $K^{+} \Lambda$ photoproduction data, which consist of nearly 3500 data points. Two different models were proposed. Both models use the same resonance configuration as described above, but in model A [B] the mass and width of the $N^{*}(2080) D_{13}\left[N^{*}(1900) P_{13}\right]$ resonance were considered as free parameters. A more detailed discussion of both models can be found in Ref. [9]. In the present discussion we use model A, since it fits the experimental data better than model B.

\section{Result for the Narrow Resonance}

By using the first isobar model the existence of a narrow resonance in kaon photoproduction was investigated by scanning the changes of the $\chi^{2}$ (indicated by the $\Delta \chi^{2}$ ) after the inclusion of this resonance in the energy range between 1620 and $1730 \mathrm{MeV}$ [6]. One of the results of this scanning process is shown in Fig. 2, where in this case the total width is taken from 1 to $10 \mathrm{MeV}$ (indicated by 10 almost overlapping lines) and the $K \Lambda$ total branching ratio is 0.2 . Figure 2 clearly shows that there are three possible narrow resonances located at $m_{N^{*}}=1650,1700$, and $1720 \mathrm{MeV}$. Nevertheless, the most convincing one seems to appear at $m_{N^{*}}=1650 \mathrm{MeV}$, where the lowest $\Delta \chi^{2}$ is obtained for $\Gamma_{N^{*}}=5 \mathrm{MeV}$. Clearly, this is not the same narrow resonance found in the $\eta$ photoproduction off a free neutron [4], where the corresponding mass is $1680 \mathrm{MeV}$. Nevertheless, this finding corroborates the result of the topological soliton model of Walliser and Kopeliovich [12] as well as that of the $\chi$ QSM model of Diakonov and Petrov [3].

We have investigated the origin of the minimum at $m_{N^{*}}=1650$ shown in Fig. 22 and found that it comes from the recoiled $\Lambda$ polarization as displayed in the left panel of Fig. 3. Comparison with experimental data indicates that the minimum appears in all available $\theta$ bins. The existence of this minimum creates a valley structure in the recoiled polarization if this observable is presented in a three-dimensional plot as shown in the right panel of Fig. 3. Despite showing the clear structures at $1650 \mathrm{MeV}$, the present experimental data have error bars that are comparable to the structures themselves. Therefore, it is obviously very important to refine these data in the future, especially 

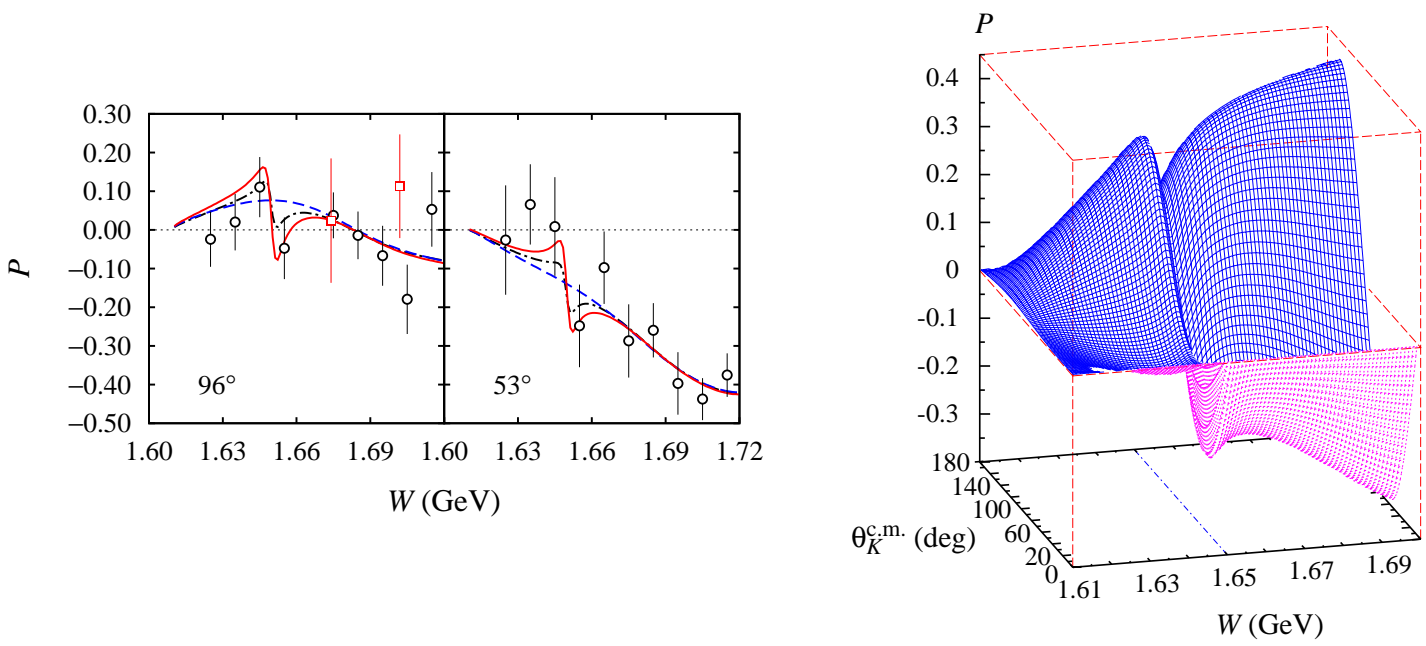

Figure 3: (Left Panel) The recoiled $\Lambda$ polarization calculated from the models with a $P_{11}$ (solid line) and an $S_{11}$ (dash-dotted line) narrow resonances compared to that without a narrow resonance (dashed line) and experimental data [13]. (Right Panel) Same as in the left panel, but presented in a three-dimensional plot.

at $W$ around $1650 \mathrm{MeV}$. This could become an important agenda of the Crystal Ball Collaboration at MAMI, Mainz.

The possibility of other quantum states, i.e. the $J^{p}=1 / 2^{-}\left(S_{11}\right)$ and $3 / 2^{+}\left(P_{13}\right)$, is ruled out by the fact that both states produce a clear structure in the total cross section, which is not observed experimentally 帆].

\section{Increasing the Resonance Width}

The total width found in the previous Section, $\Gamma_{N^{*}}=5 \mathrm{MeV}$, provides an evidence that the resonance is narrow. However, as discussed above, this resonance is certainly different from that observed by Kuznetsov et al. [ $\left[\right.$ ] at $m_{N^{*}}=1680 \mathrm{MeV}$. Based on this fact it is obviously interesting to study the effect of extending the upper limit of the resonance width in our previous investigation [6]. Moreover, in our previous study there is also a possibility for a resonance with a mass around $1680 \mathrm{MeV}$, although the corresponding signal is slightly weaker. By scanning the total width up to $100 \mathrm{MeV}$ we found an absolute minimum of $\Delta \chi^{2}$ as shown in the left panel of Fig. $甘$ 河. At this point we obtain $m_{N^{*}}=1696 \mathrm{MeV}$ and $\Gamma_{N^{*}}=76 \mathrm{MeV}$. Note that we still observe the minimum at $m_{N^{*}}=1650 \mathrm{MeV}$, which corresponds to the narrow resonance found in the previous study, although we have used a fully covariant isobar model [9] to obtain this result. Interestingly, the signal at 1650 $\mathrm{MeV}$ vanishes as we increase the width beyond $25 \mathrm{MeV}$.

In case of $\Gamma_{N^{*}}>25 \mathrm{MeV}$ we can observe a single minimum of $\Delta \chi^{2}$ for each values of $\Gamma_{N^{*}}$. Actually, this is seen in the left panel of Fig. $\sharp$. By plotting this minimum value as a function of the resonance width we arrive at the resonance mass and width relation depicted in the right panel of Fig. \#, where we have added the mass uncertainty obtained from the MINUIT code. Obviously, the mass is bounded between 1675 and $1695 \mathrm{MeV}$, which is consistent with the PDG estimate [5]. However, by considering the coupling constant value of the $N^{*}(1710) P_{11}$ resonance, which should not exceed that of the $N^{*}(1650) S_{11}$, our finding suggests that $\Gamma_{N^{*}} \lesssim 35 \mathrm{MeV}$, which, according to 

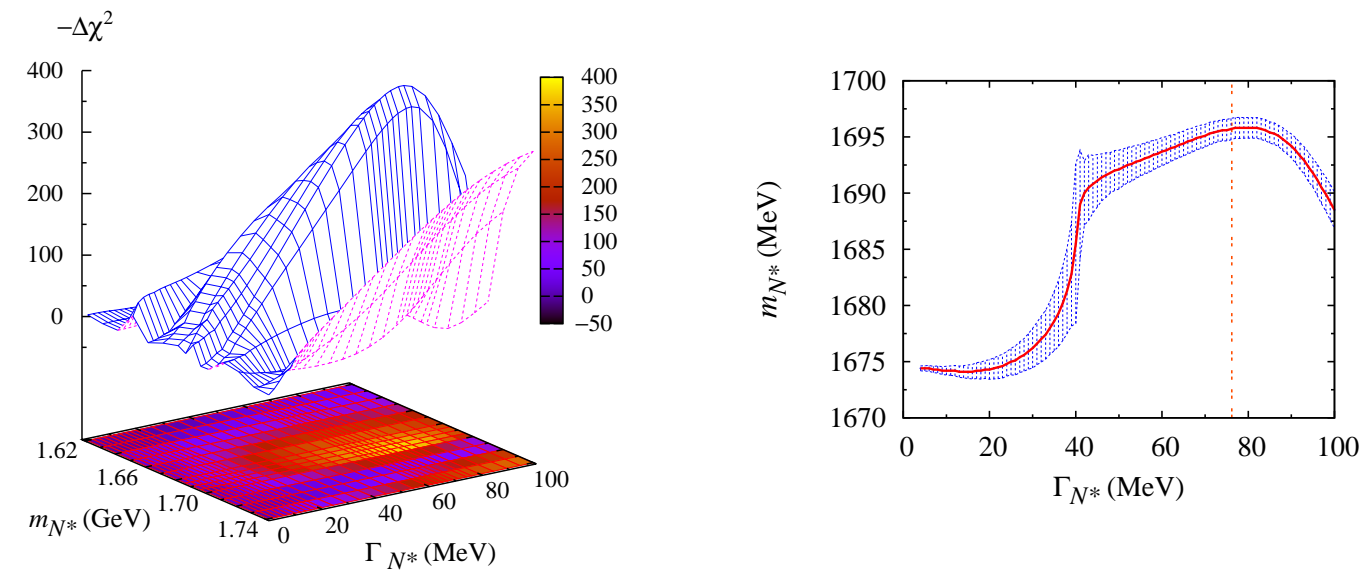

Figure 4: (Left Panel) Same as in Fig. 2, but obtained by using the covariant isobar model [9] and displayed in three-dimensional plot as functions of the resonance mass and total width. (Right Panel) Relation between the resonance mass and total width 沛].

the mass and width relation of Fig. 4, corresponds to $m_{N^{*}} \lesssim 1680 \mathrm{MeV}$ [7]. This result is much closer to the PDG estimate of the $N^{*}(1685)$ resonance [5].

\section{References}

[1] D. Diakonov, V. Petrov, and M. Polyakov, Z. Phys. A 359, 305 (1997).

[2] R. M. Barnett et al. [Particle Data Group], Phys. Rev. D 54, 1 (1996).

[3] D. Diakonov and V. Petrov, Phys. Rev. D 69, 094011 (2004).

[4] V. Kuznetsov et al., Phys. Lett. B 647, 23 (2007); Phys. Rev. C 83, 022201 (2011).

[5] J. Beringer et al. [Particle Data Group], Phys. Rev. D 86, 010001 (2012).

[6] T. Mart, Phys. Rev. D 83, 094015 (2011).

[7] T. Mart, Phys. Rev. D 88, 057501 (2013).

[8] T. Mart, Phys. Rev. C 82, 025209 (2010).

[9] T. Mart and M. J. Kholili, Phys. Rev. C 86, 022201(R) (2012).

[10] F. X. Lee, T. Mart, C. Bennhold, H. Haberzettl, and L. E. Wright, Nucl. Phys. A 695, 237 (2001).

[11] H. Haberzettl, C. Bennhold, T. Mart and T. Feuster, Phys. Rev. C 58, R40 (1998).

[12] H. Walliser and V. B. Kopeliovich, Zh. Eksp. Teor. Fiz. 124, 483 (2003).

[13] R. Bradford et al., Phys. Rev. C 73, 035202 (2006); M.E. McCracken et al., ibid. 81, 025201 (2010). 